\title{
Infrared Deflectometry for Slope Deformation Measurements
}

\author{
H. Toniuc ${ }^{1} \cdot$ F. Pierron ${ }^{1}$ \\ Received: 15 November 2018 / Accepted: 3 February 2019 / Published online: 17 July 2019 \\ (C) The Author(s) 2019
}

\begin{abstract}
This paper presents an implementation of deflectometry in the infrared spectrum. Deflectometry consists in recording the specular image of a reference grid pattern onto the mirror-like surface of a test specimen. This technique has two main advantages, high sensitivity and direct measurement of surface slopes, which in the case of thin plate bending is only one spatial differentiation away from surface strains. The objective of imaging in the infrared spectrum is to mitigate the main limitation of deflectometry in the visible spectrum, which is to require an extremely smooth surface to provide dominant specular reflection. This paper explores IR deflectometry for the first time for deformation measurements. Two different infrared cameras were assessed for use in IR deflectometry, a short wave quantum detector one, and a long wave microbolometer (MB) array one. Different materials of varying surface roughness were imaged and it was verified that the Rayleigh criterion was appropriate to determine whether IR deflectometry was feasible on a given surface. With the MB camera, most off-the-shelf material surfaces proved reflective enough to perform IR deflectometry. Finally, several bending tests were performed on aluminium plates and the deformation fields were shown to compare remarkably well with finite element simulations. The experimental data were then used in the Virtual Fields Method (VFM) and the elastic stiffness components of aluminium were retrieved with excellent accuracy, further validating IR deflectometry.
\end{abstract}

Keywords Deflectometry · Full-field measurement · Infrared imaging · Grid method · Slope measurement

\section{Introduction and State of the Art}

Deflectometry is a technique that measures surface slopes of solids by analysing the specular reflection image of a target. There are three main applications: shape measurement of mirror-like solids like wind-shields or car bodies, defect detection and measurement of deformation of solids. The latter is the focus of this article, the reader is referred to [1] and [2-4] for the two former, respectively.

The first mention of deflectometry for deformation measurements dates back to Ligtenberg in 1954 [5]. The pattern was a grid and moiré fringe analysis was used to determine surface slopes on a flat plate, in normal incidence configuration, i.e. the camera axis is perpendicular to the

\section{F. Pierron}

F.Pierron@soton.ac.uk

H. Toniuc

horea.toniuc@gmail.com

1 Mechanical Engineering, Faculty of Engineering and Physical Sciences, University of Southampton, University Road, Southampton SO17 1BJ, UK plate. Variations of this technique were published over the years under the denomination 'reflection (or reflective) moiré' [6-10] though it never attracted much attention outside a small group of researchers in the experimental mechanics community.

The data processing of the slope measurement has evolved with the advent of digital vision and computerized image processing, though the configuration has remained more or less the same [11-13]. It is worth noting that different names have been used over the years, for instance [13] refers to it as 'full-field digital gradient sensing', but the name 'deflectometry' is the most widespread in the shape measurement community, by far the largest community working on it, and so the use of this denomination is strongly encouraged by the present authors to avoid confusion. The processing of the grid images to derive surface slopes is generally performed through phase shifting (also called phase stepping), with two different approaches depending on the problem to be solved. Temporal phase shifting describes a set of techniques where the grid pattern is moved sequentially and several images are recorded. This enables to solve for the local phase using the different grey level values at a single pixel along time [11]. The main advantage of temporal phase shifting is that there is no 
requirement for a regular grid image so this can be used on non-flat surfaces [1]. The main disadvantage however is that this process takes time and as a consequence, it cannot be applied to transient dynamic events. The alternative is called spatial phase shifting (also known as sampling moiré [14]) and consists in using adjacent pixels to perform the phase-shift $[15,16]$. This is ideal for dynamic events as all information is contained in a single image. However, the spatial resolution is reduced and it is only applicable to highly regular grid images. This means here that the panel under inspection needs to be rigorously flat.

While the previously cited studies have all used grid (or grating) patterns, it is possible to use a random pattern. In this case, a correlation algorithm is needed to detect the apparent in-plane movements of the pattern that relate to the slopes [17]. However, the advantage of using a random pattern is not clear as when the use of a grid is possible, it was shown to be superior to a random one because of the possibility to move to a more efficient phase shifting detection algorithm [18].

The main requirement for deflectometry is that the surface provides dominant specular over diffusive reflection. The main parameters that drive this are the wavelength of the light $\lambda$, the incidence angle $\theta$ of the illumination measured from the surface normal and the RMS roughness of the surface $\sigma$. A surface can be classified as specularly reflective through the Rayleigh criterion [19]:

$$
\frac{\lambda}{\sigma \cos \theta}>8
$$

In the visible spectrum, $\lambda$ is approximately $500 \mathrm{~nm}$. For the Rayleigh criterion to be met, a surface roughness smaller than $60 \mathrm{~nm}$ is required in normal incidence. This is the major current limitation of deflectometry as it can only be applied to highly-smooth surfaces and when used on as-manufactured engineering surfaces, coatings must be applied [20] which is inconvenient and currently limited to flat surfaces. If deflectometry is ever going to be used as a widespread tool to measure the deformation of surfaces in engineering, this restriction needs to be lifted. As clearly explained in [21], imaging in the infrared spectrum relaxes the requirement for a very smooth surface. In the long infrared (wavelength of $10 \mu \mathrm{m}$ ), the Rayleigh criterion relaxes to a surface roughness of $1.25 \mu \mathrm{m}$, which is compatible with many 'as-manufactured' engineering surfaces without any specific surface treatments, as will be shown later in the present article.

Infrared deflectometry has been reported sporadically in the literature for surface defect assessment. For shape measurements, if the surface is diffusive, fringe projection can be used and there is little advantage of using the more complicated deflectometry. However, deflectometry is ideal to reveal surface defects because it is sensitive to the slope. It is common knowledge that when wanting to inspect the quality of a surface, one tries to look at it at a shallow angle to enhance the specular component of the reflection that can show the surface defects.

The first application of infrared deflectometry, to the best knowledge of the present authors, appeared in 2005 in [2] with the purpose of surface defect detection. The infrared pattern was generated by sticking bands of black plastic tape onto a brushed aluminium plate. When heated up to approximately $40^{\circ} \mathrm{C}$, the difference in emissivity between the plastic (0.95) and the aluminium (0.3) produced a contrasted pattern which reflection on an unpainted car body part could be recorded with an infrared camera operating in the long wave spectrum. Unidirectional grid lines only were used in this application, and temporal phase shifting was used to retrieve the phase information. Defects could thus be clearly identified on the studied part. Later, Sarosi et al. [22] attempted to use different types of displays to perform temporal phase shifting but with limited success. The difficulty to achieve a screen-based infrared pattern was later confirmed by [21] where only resistor-based arrays were identified as an alternative. Scanning a laser across a screen was attempted $[21,23]$ but with the drawback of pattern blurring by heat diffusion and a longer response time. A heated metal ribbon was also used to control mirror surfaces [24]. However, the most promising route is clearly a static pattern as in [2], as confirmed in [21].

In spite of the potential of infrared deflectometry, only few references exist on the subject, as shown above, and all concern defect detection. Infrared deflectometry has never been demonstrated to measure deformations, and has never been used with spatial phase shifting. Also, none of the references above have studied performance evaluation of the camera sensors in terms of random noise and how it affects the measurements. The objective of the present article is to address these points and demonstrate that infrared deflectometry can become a new useful item in the experimental mechanics toolbox.

The paper is organized around three main sections. First, the performances of two infrared cameras are reviewed in terms of grey level and phase noise; then applicability is demonstrated over a range of as-manufactured materials surfaces for metals, polymers and glass. Finally, bending tests have been performed on aluminium plates and the results were validated by comparing the full-field curvatures to the data from a finite element model. The Virtual Fields Method was also employed to identify the elastic stiffness components and compare the values to the known aluminium Young's modulus and Poisson's ratio, providing further validation. 
Table 1 Specifications of the two infrared cameras used in this study

\begin{tabular}{lll}
\hline Camera & FLIR SC7600 (QD) [25] & FLIR A655SC 25 $(\mathrm{MB})[26]$ \\
\hline Waveband $(\mu \mathrm{m})$ & MW $(1.5-5.1)$ & LW $(7.5-14)$ \\
Detector type & InSb & Micro-bolometer array \\
& & VOx (Vanadium Oxide) \\
Pixel resolution & $640 \times 512$ & $640 \times 480$ \\
Detector pitch $(\mu \mathrm{m})$ & 15 & 17 \\
Temperature range $\left({ }^{\circ} \mathrm{C}\right)$ & up to 300 & -40 to 150 \\
Integration time $(\mu \mathrm{s})$ & $0.1-20,000(1,800$ here $)$ & - \\
Time constant $(\mathrm{ms})$ & - & 8 \\
Max. frame rate $(\mathrm{Hz})$ & 100 & 50 \\
f-number $($ with lens $)$ & $\mathrm{f} / 3$ & $\mathrm{f} / 1$ \\
NETD & $<20 \mathrm{mK}$ & $<30 \mathrm{mK}$ \\
Lens focal distance $(\mathrm{mm})$ & 25 & 24.6 \\
Field of view & $22^{\circ} \times 17^{\circ}$ & $25^{\circ} \times 19^{\circ}$ \\
\hline
\end{tabular}

\section{Experimental Elements}

\section{Infrared Cameras}

Two infrared cameras have been used in this study. The first one is a FLIR SC7600 camera [25] with InSb quantum detector (later referred to as QD camera), and the second one is a FLIR A655SC $25^{\circ}$ camera [26] with microbolometer detectors (later referred to as MB camera). The performances of both systems are recorded in Table 1 . The objective here is to check whether the performances of the MB camera could come close to that of the QD one as the former is typically an order of magnitude less expensive than the latter. Also, for future development, some MB cameras are currently available with higher spatial resolutions and are likely to develop further in that direction because of the thermal surveying market. The other interest of this comparison here is that the QD camera operates in the short infrared while the MB records in the long infrared (see Table 1), so that the Rayleigh criterion can be checked in practice for two typical IR cameras. Finally, the MB camera has a 'noise reduction' feature which most probably consists in averaging out pixels spatially. It is important that this feature is turned off in the camera software as it creates spurious image defects, probably from the presence of defective pixels that get amplified by spatial averaging.

\section{Infrared Grid Pattern Generation}

As detailed in [21] and reported in the introduction of this paper, there are different ways to generate an infrared pattern. Among the preferred options are the use of an emissivity-contrasted pattern that is heated up. In [2], black plastic tape fixed onto a brushed aluminium plate was used. They report the emissivity of the aluminium to be about $0.1-0.3$, and that of the tape to be 0.95 . The panel is then heated up to $313 \mathrm{~K}$, which according to the authors provides maximal emission at a wavelength of $9.3 \mu \mathrm{m},{ }^{1}$ which is ideal for the MB camera. The same idea has been used here, but since a bi-directional grid had to be used, black squares were directly printed onto $1.5 \mathrm{~mm}$ thick brushed aluminium plates, according to the procedure reported in [28, 29]. Nominal grid pitches of 2.0 and $7.14 \mathrm{~mm}$ were used in this study for the noise and fill factor assessment on one side, and bending tests on the other, respectively. The plates dimensions were $300 \times 250 \mathrm{~mm}^{2}$ for the $2 \mathrm{~mm}$ pitch grid and $700 \times 1000 \mathrm{~mm}^{2}$ for the $7.14 \mathrm{~mm}$ pitch one. A photograph of the $7.14 \mathrm{~mm}$ pitch grid is shown in Fig. 1. The grid is then heated up using a hair dryer up to a temperature on the order of $50^{\circ} \mathrm{C}$ before measurements can be made. The convection is sufficiently low in the small test lab that the plate remains at this temperature for the time it takes to perform the experiments, unless otherwise stated in the text. Thanks to the high diffusivity of the aluminium grid plate, spatial uniformity of the grid was achieved well within $1{ }^{\circ} \mathrm{C}$, though this is not very critical here. In the future, it is envisaged to use adhesive heating sheets such as described in [30] to ensure thermal stability and temperature control over longer periods of time. It should be noted that it was also attempted to print black lines to invert the contrast when heated up. The results are not shown in this paper as they were very similar to that obtained with the black dot grid but the images and related data are available in the data repository.

\section{The Grid Method}

The Grid Method (GM) is an alternative to Digital Image Correlation relying on imaging a regular grid rather than a random pattern. In this case, it has been shown that spatial

\footnotetext{
${ }^{1}$ According to Wien's displacement law [27]
} 


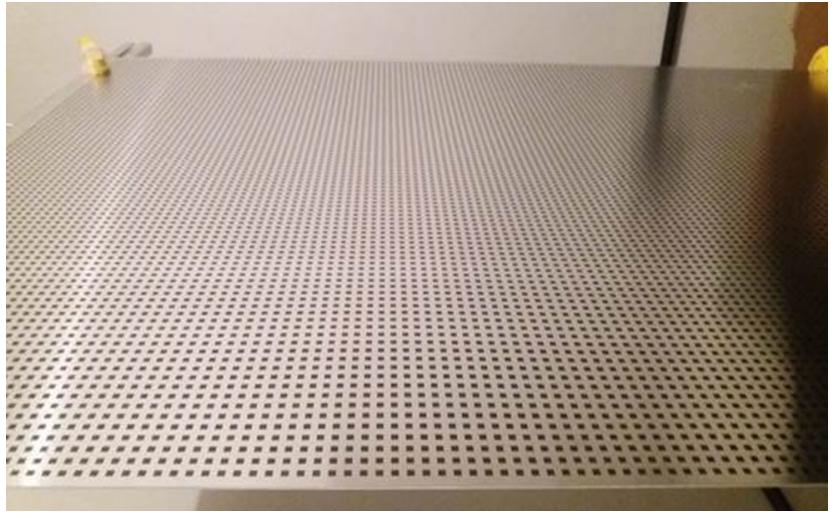

Fig. 1 Printed grid, pitch of $7.14 \mathrm{~mm}$

phase shifting (or phase stepping) is the optimal way to solve the image correlation problem between deformed and undeformed states [18]. Different versions of the spatial phase shifting algorithm have been proposed, as reviewed in [16]. Here, the standard Windowed Discrete Fourier Transform (WDFT) algorithm was used with a triangular window. This has been used extensively in the past and the procedure is not recalled here. The reader is referred to [16] for all details. The related Matlab programs are also available in the data repository.

\section{Camera Performance Assessment}

\section{Sampling and Grid Quality}

The first step is to verify the grid quality. The heated reference grid plate, similar to that represented in Fig. 1 but with a grid pitch of $2 \mathrm{~mm}$, has been directly imaged with both cameras, with camera axis perpendicular to the plate. The grid images were processed to obtain phase, while keeping only one data point per grid pitch. If the grid and the lens were perfect and if the sampling (number of pixels per period) were exactly integer, then the phase map would be perfectly uniform. ${ }^{2}$ In practice, lens distortion will create a low spatial frequency distortion of the image, and poor grid quality may add fringing on the phase maps. The latter however may not be very apparent on the phase maps themselves but rather on their derivatives. This is the procedure that has been adopted here to check for grid and sampling quality for both cameras. The procedure is detailed in the flowchart of Fig. 2.

The first step is to adjust the magnification so that the pixel sampling of the grid is the closest possible to an integer value. Here, a value of $\mathrm{N}=5$ pixels per period was selected which is common for the GM. However, when a value close

$\overline{{ }^{2} \text { Only rigorously true for a telecentric lens }}$

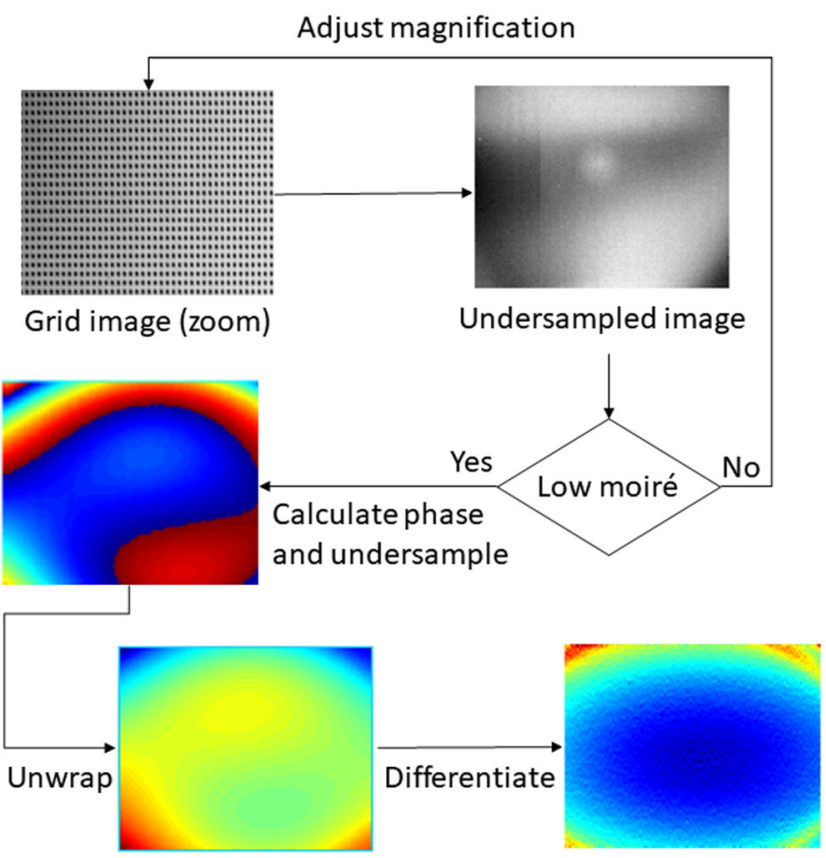

Fig. 2 Flowchart for grid image quality assessment

to $\mathrm{N}=5$ is reached, it is necessary to adjust the magnification around this position to get the closest possible to exactly 5. To do this, an undersampled image is used. Again, if the grid and lens were perfect, keeping only one pixel in 5 would lead to a uniform image. When the sampling is too far from $\mathrm{N}=5$, moiré fringes appear in the downsampled image. Figure 3 shows the two downsampled images obtained for both cameras on the same grid. The MB camera image only shows one vertical moiré fringe while horizontally, less than one fringe is present. This is excellent sampling, most of the contrast comes from the lens distortion. For the QD camera, more fringes can be seen and their concentric circular patterns illustrates the fact that the lens on the QD camera exhibited higher levels of distortion.

Now that the sampling has been adjusted to avoid spurious phase fringing arising from improper grid sampling, the phase maps can be calculated, unwrapped and differentiated as detailed in Fig. 2. It is worth noting that the phase maps, once obtained from the full image, are downsampled by keeping only one pixel per grid period. These phase maps
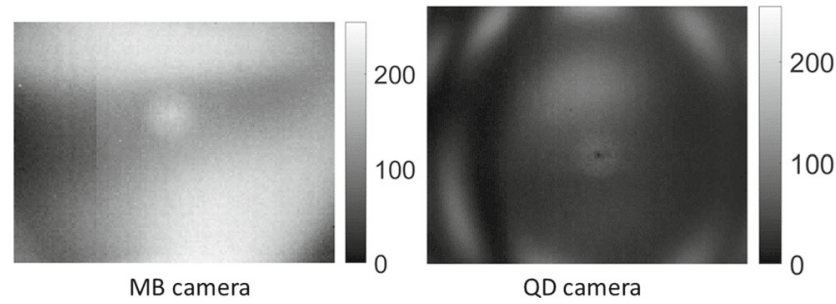

Fig. 3 Undersampled images of the grids keeping only one pixel over 5 , scale in grey levels 

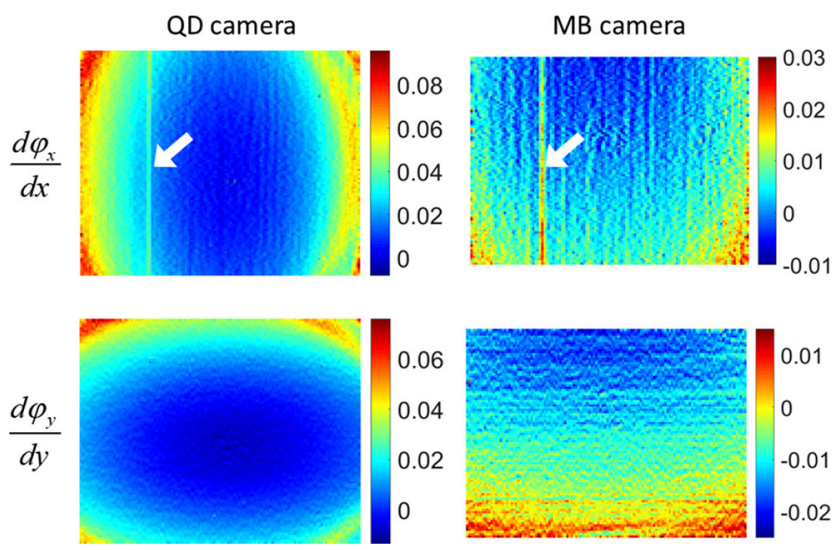

Fig. 4 Phase derivative maps for both cameras, in dimensionless units; white arrow pointing at consequence of grid defect

are scaled in pixels by multiplying the unwrapped phase by $\mathrm{N} / 2 \pi$, and then differentiated using a step of $\mathrm{N}$. The resulting maps are directly expressed as 'strain' as if the image were a deformed one from a perfect reference. For a perfect grid image, this 'strain' should be exactly zero so any grid/lens quality issue can be directly assessed and compared across different images or imaging systems. Here, the objective is to compare the two cameras. The phase derivative maps are shown in Fig. 4. A significant grid irregularity issue would show fringes along $\mathrm{x}$ or $\mathrm{y}$. One can see such an irregularity on the derivatives of the $\mathrm{x}$ phase maps for both cameras. This is thought to be a single grid defect most probably arising from the printing as suggested in [28], though this would need to be confirmed. However, apart from this, no other fringing can be seen, confirming the good quality of the grid. When working differentially, such grid defect will cancel out, even more so when the iterative phase extraction method is used, as reported in [16]. Finally, what can also be seen in these plots is the large distortion arising from the lens used with the QD camera, the scale of the phase derivative being about three times that of the MB image. The impression of larger random noise may also be caused by the smaller scale of the MB images. The next section is dedicated to investigating the effect of random noise.

\section{Noise}

The effect of random camera noise has to be investigated as it has a significant effect on the quality of the deformation measurements. To do so, the same setup as above has been employed. The grid plate was first heated up to approximately $50^{\circ} \mathrm{C}$ and then, 20 series of 10 images were recorded at $50 \mathrm{~Hz}$ for the $\mathrm{BD}$ camera and $60 \mathrm{~Hz}$ for the QD camera (with an integration time of $1800 \mu \mathrm{s}$ ). Between each series of 10 images, a waiting time of $30 \mathrm{~s}$ was observed to allow for the reference grid to cool down so that over the 20 series of 10 images, the effect of the reference grid temperature could be evaluated. Because of the presence of the reflection of the camera lens at the centre of the image, as the temperature decreased, saturation occurred, see Fig. 5. The data was therefore processed after masking off this central part of the images. Some more images were gathered at an angle to avoid this problem and provided the same trend for the noise. The full set of images and data is available in the attached data repository.

\section{Grey level noise}

For each series of tests, images were subtracted two by two, i.e. image 2 - image 1, image 4 - image 3 etc. This resulted in grey level noise images, as shown in Fig. 6. The noise is expressed as a fraction of the dynamic range, here, 256 grey levels as all images have been exported in 8-bit tiff format. As a point of reference, typical machine vision CCD cameras exhibit noise values between 0.5 and $1 \%$ depending on the imaging conditions. Also, for such cameras, the noise maps are generally random, only specific ultra-high speed cameras sometimes exhibit spatially correlated noise [31]. It can be observed that while the MB camera exhibits a random noise map, the QD does not and the pattern of the grid is still apparent. The reason for this is that the noise is not homoscedastic. This can be seen in Fig. 7. To create these plots, the first series of 10 images were considered and the first image of the series has been subtracted to all others. This provided a series of 9 noise maps from which the histogram of the noise standard deviation was established. This was performed using the noise evaluation tool in the MatchID software [32]. The noise is purely homoscedastic for the MB camera, while an unusual heteroscedastic distribution is visible for the QD camera, with maximum variance for intermediate grey levels, explaining the pattern in Fig. 6. It should be recalled here that for machine vision CCD cameras, the noise is also heteroscedastic but contrary to the evolution observed for the QD camera, the variance increases linearly and monotonically from low to high grey level values (Fig. 2d) in [33]).

The 20 series of images were processed to evaluate the standard deviation of noise as a function of plate temperature. This is represented in Fig. 8. The standard deviation was calculated as the average over each series. It can be seen that there is a very strong variation of the noise for the MB camera, while the QD camera exhibits very little variation. This indicates that the reference plate temperature has to be much more carefully selected when measurements are performed using the MB camera. It has not been possible to increase the temperature further with the hair dryer. It should be possible to go much further in temperature using the wire wound flexible heaters from [30] but higher temperatures may create spurious image distortions because 

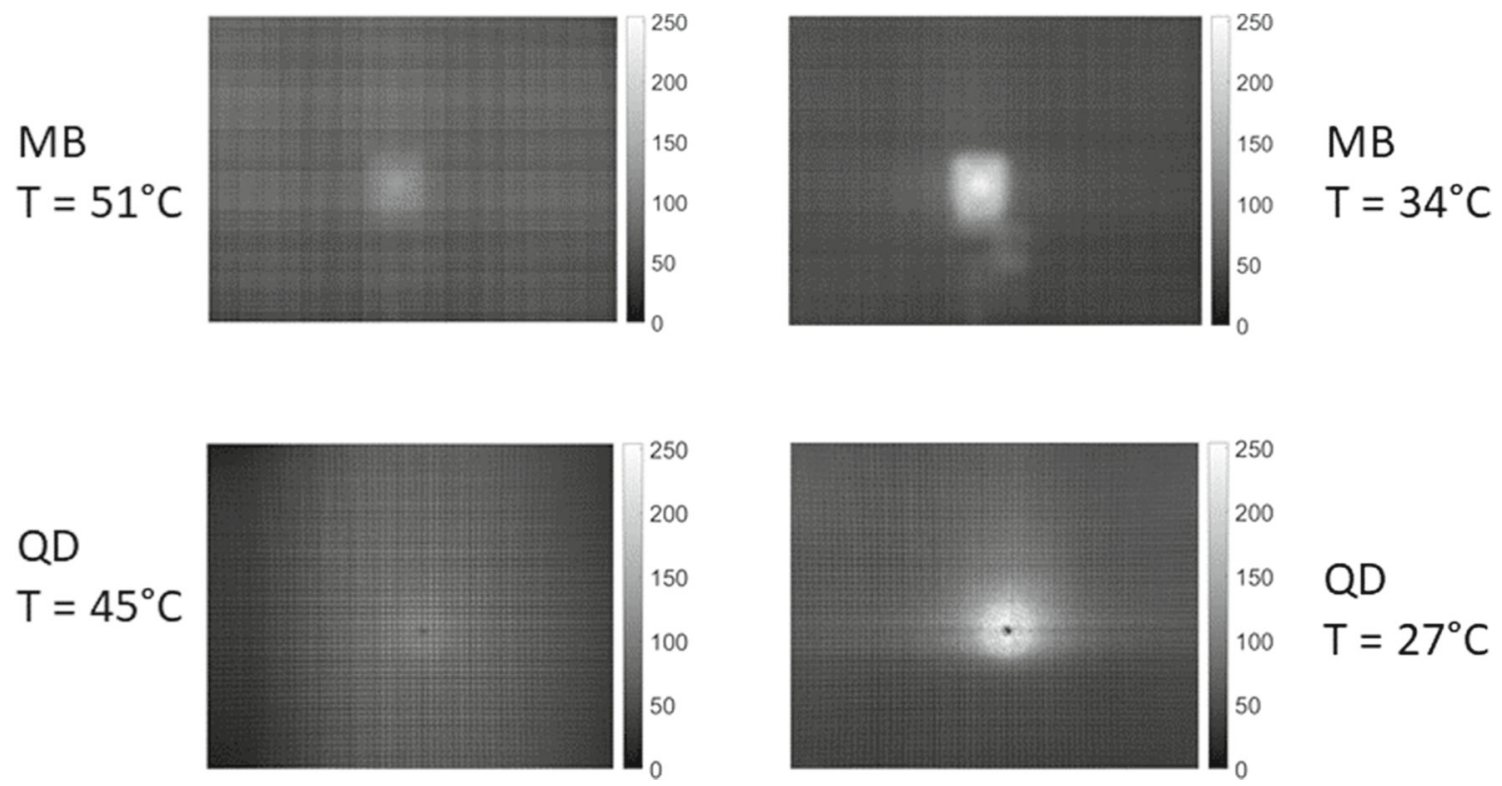

Fig. 5 Illustration of the hotspot caused by the reflection of the IR camera on the test plate
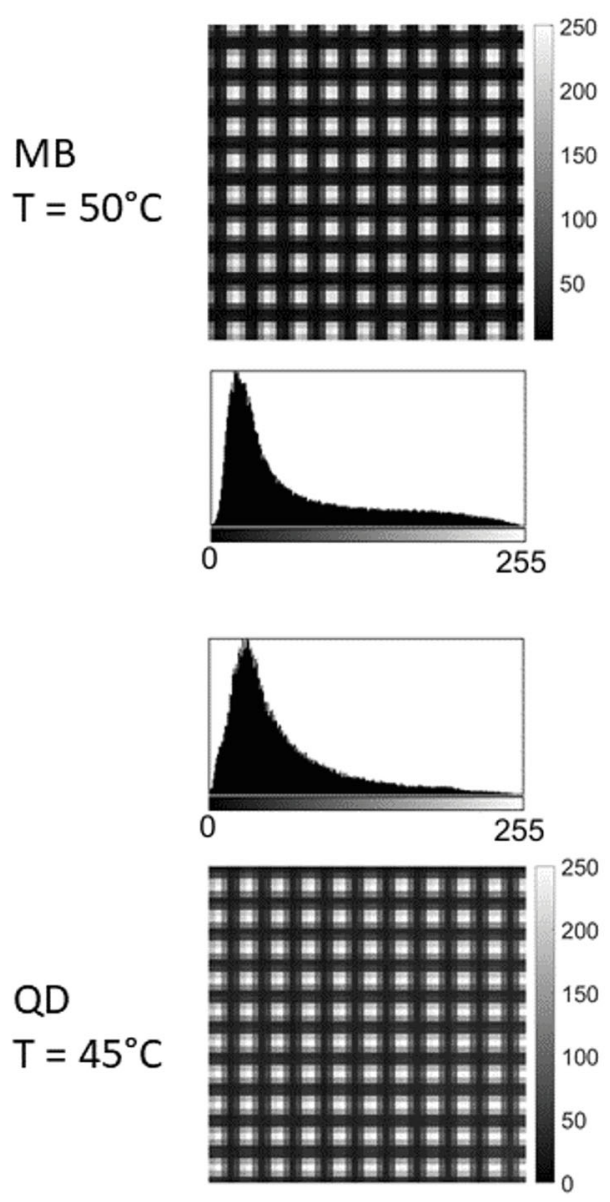

Fig. 6 Noise evaluation procedure
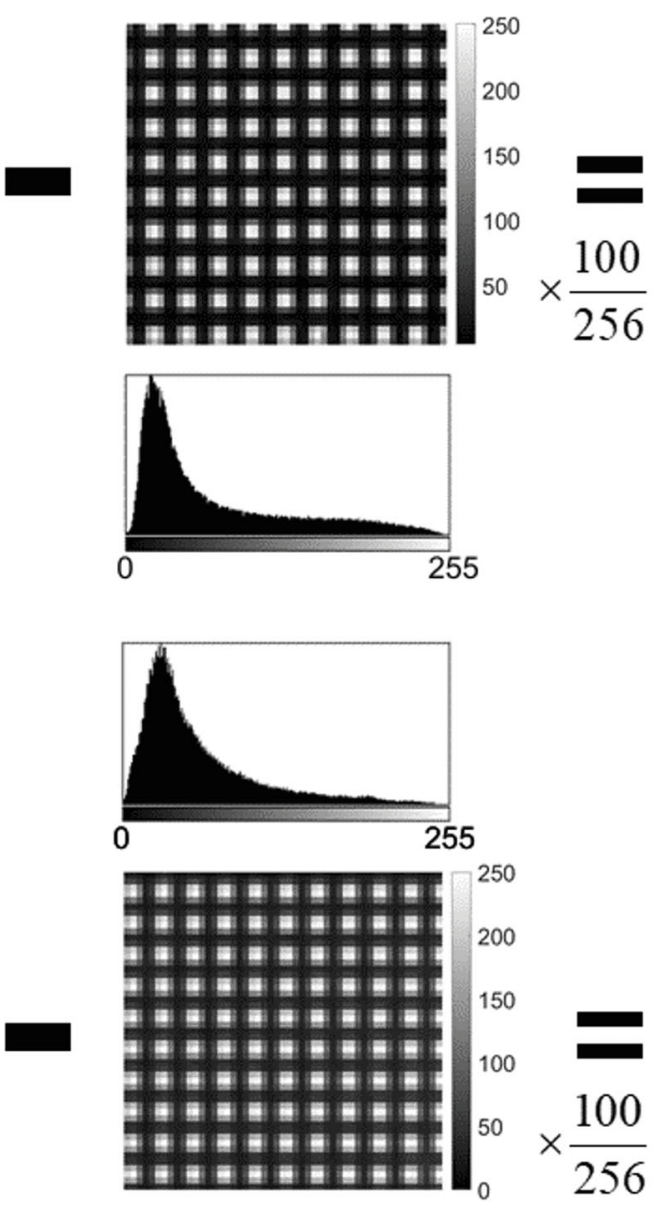
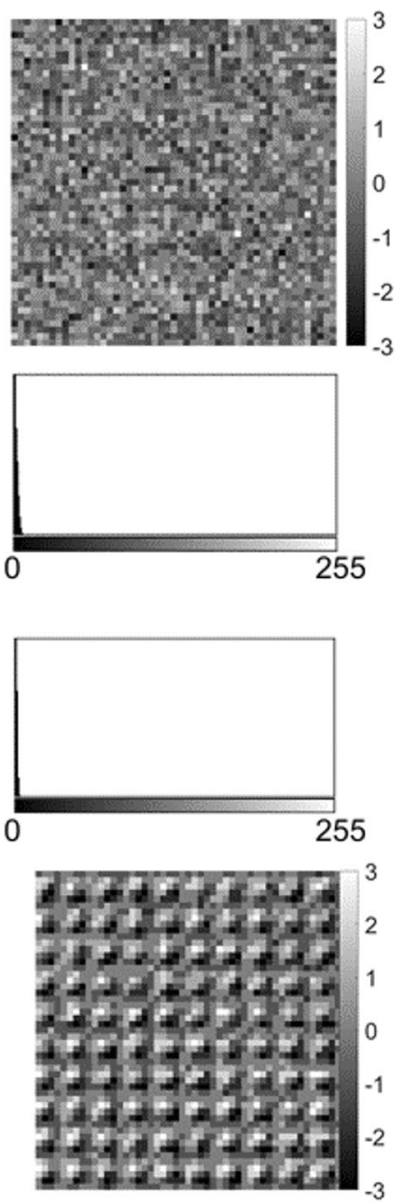

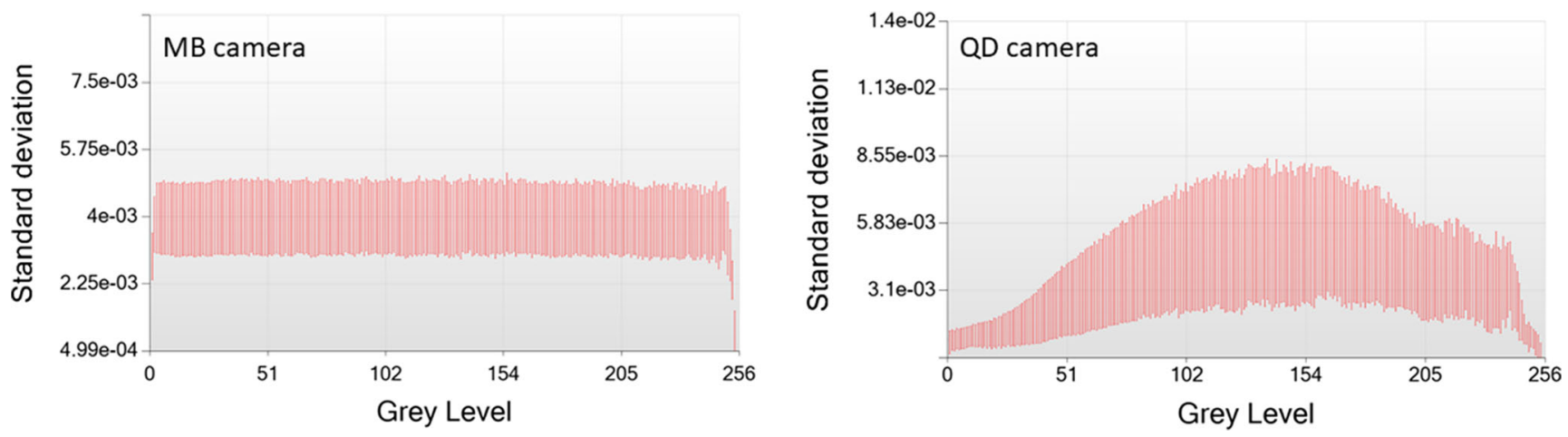

Fig. 7 Variation of the noise standard deviation (in pixel) as a function of grey level for both cameras

of hot air convection between the camera and the specimen. In any case, the grey level noise at $50^{\circ} \mathrm{C}$ for the $\mathrm{MB}$ camera is comparable to that of a machine vision CCD camera and therefore, good quality measurements should be achievable. It should also be noted here that the start temperature for the QD camera was lower than for the MB camera. This is a consequence of the relative lack of control of the heating up with the air blower, as well as variation in the room temperature. The QD tests were performed in a different room with cooler temperature $\left(21 / 22^{\circ} \mathrm{C}\right)$ than that in which the MB experiments were performed (a warmer dark room, in the summer, $26 / 27^{\circ} \mathrm{C}$ ). The range of temperature is the same however as the plates were left to cool down for the same amount of time in both cases.

\section{Phase/slope noise}

The effect of the above camera noise on phase noise has been evaluated from the series of images reported above. The phase was calculated incrementally between image 1 and image 2, image 3 and image 4 etc, and the central part of the images was cropped out as for the grey level noise since phase maps there were largely spurious because of

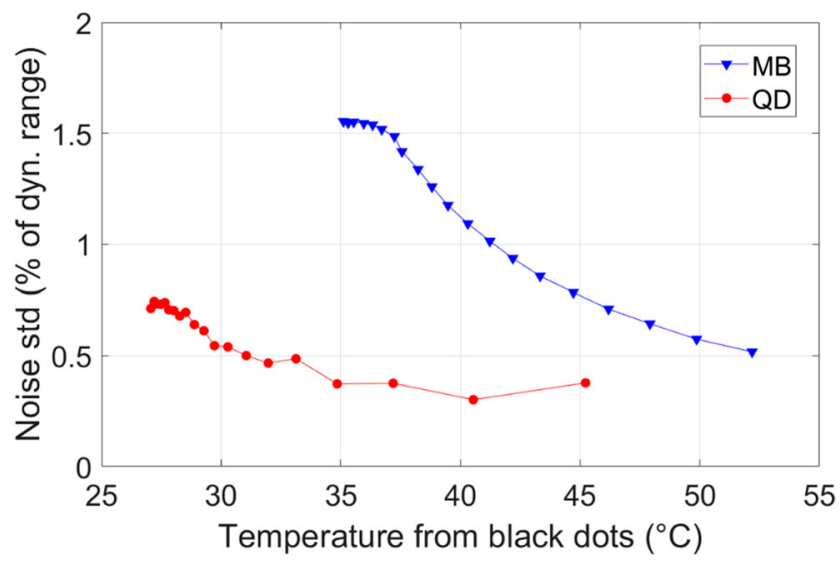

Fig. 8 Standard deviation of grey level noise maps expressed as percentage of the dynamic range, as a function of the plate temperature the saturation. The standard deviation of each phase noise map was calculated and averaged over the 5 noise maps for each temperature, again keeping only one data point per grid pitch. The phase was then expressed in pixel by multiplying it by $5 / 2 \pi$, so that the values could directly be compared to a displacement resolution for the in-plane grid method or digital image correlation. The same data was also expressed as physical slope by multiplying the phase by $\mathrm{p} / 4 \pi \mathrm{h}$ where $\mathrm{h}$ is the specimen to grid distance, here, $560 \mathrm{~mm}$ and $\mathrm{p}$ is the grid pitch, here, $2 \mathrm{~mm}$. The results are reported in Fig. 9. It must be noted here that this $h$ value simulates the slope resolution attained if the images were that of an actual grid reflection experiment with a stand-off distance of $h$. One can see that as expected, the phase noise follows the same trend as the grey level noise, though the differences are somewhat more marked between high and low temperature. At temperatures above $45^{\circ} \mathrm{C}$, both cameras provide measurement uncertainties better than 0.01 pixel (i.e. $1 / 500$ of the grid pitch) which is similar to what is obtained with standard CCD cameras with either the grid method or digital image correlation. In terms of slope, the best resolution for the MB camera is $3.6 \mu \mathrm{rad}$ (or mm.km ${ }^{-1}$ ). Again, this is comparable to what has been obtained in the visible range, though larger as the imaging distance is only $560 \mathrm{~mm}$ while $1 \mathrm{~m}$ was used in [34] for

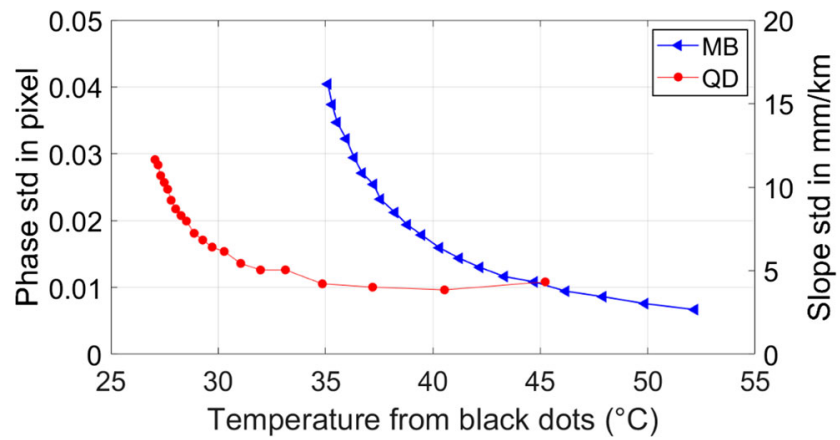

Fig. 9 Standard deviation of the phase noise expressed in pixels and slope noise in mm. $\mathrm{km}^{-1}$ as a function of the temperature, for both cameras 
Fig. 10 Maps of curvatures (in $\mathrm{km}^{-1}$ ) from stationary images at $52^{\circ} \mathrm{C}$ with the $\mathrm{MB}$ camera, with standard deviations
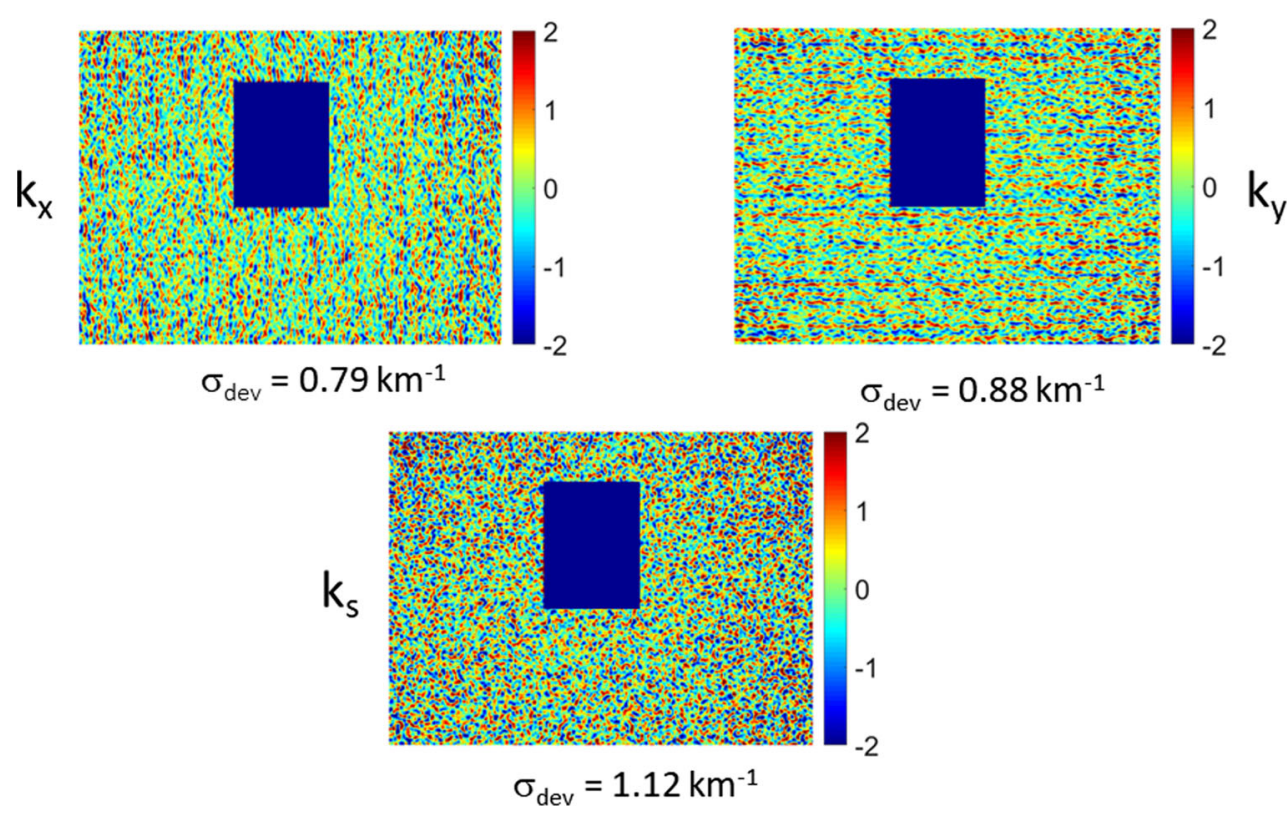

instance. For the $1 \mathrm{~m}$ imaging distance used for the bending test, this slope resolution will be less that $2 \mu \mathrm{rad}$, which is typical for this kind of experiment. This demonstrates that the current setup delivers good quality grid images that should generate quality measurements.

\section{Curvature noise}

Finally, the resolution in curvature has been studied. Indeed, when testing thin plates, the surface deformation is proportional to the surface curvatures and so, this is the relevant quantity. Curvatures have been calculated from slopes using centred finite differences. Figure 10 shows maps of curvatures from the MB images taken at the highest temperature, $52^{\circ} \mathrm{C}$. One can see spatially uncorrelated noise as expected.

The curvature standard deviation from stationary images was then calculated for all series of images. The data was also expressed in terms of surface strains by multiplying

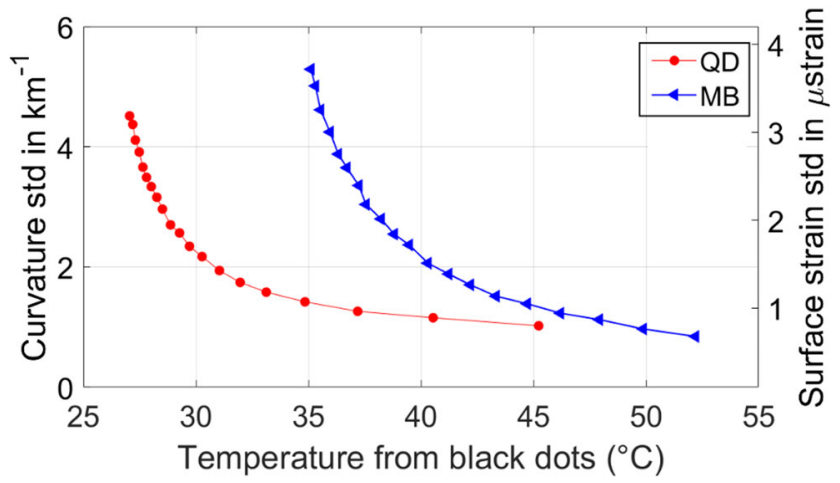

Fig. 11 Curvatures (in $\mathrm{km}^{-1}$ ) and equivalent surface strains (in $\mu$ strain) as a function of the temperature, for both cameras the curvature by $0.75 \mathrm{~mm}$ (half the thickness of the plate tested in the bending test section). The results are provided in Fig. 11. They are typical for the technique. It is worth noting the very high sensitivity of the method arising from the fact that grid to specimen distance acts as a geometric sensitivity amplification. Surface strain resolution is similar to that of strain gauges, with exceptional spatial resolution as no spatial smoothing was performed to differentiate the slopes.

\section{Exploration of Applicability}

The objective of this section is to explore the applicability of IR deflectometry on a number of materials with different emissivity and surface roughness, and to check whether the Rayleigh criterion is an effective tool to predict the applicability of IR deflectometry.

To investigate the range of possible materials to study with IR deflectometry, a number of different materials were studied. They were sourced from our workshop and commercial suppliers and have been tested 'as received' unless otherwise stated. Their roughness was measured using a Taylor Hobson Talysurf 120L. Ten measurements were performed on each sample, five in each direction, and the reported RMS roughness in Fig. 12 is the average of these ten measurements. Their typical emissivities, $\varepsilon$, were found in textbooks for ABS [35], aluminium [36], brass [37, 38], carbon/epoxy composites [39], stainless steel [37] and steel $[36,38]$. Only the PMMA, PVC and PP plates were not measured for roughness. This is because these tests were not performed at the same time as the others and had for objective to check whether IR deflectometry could be used 
Fig. 12 Reflected grids imaged with the MB camera on a set of typical engineering materials

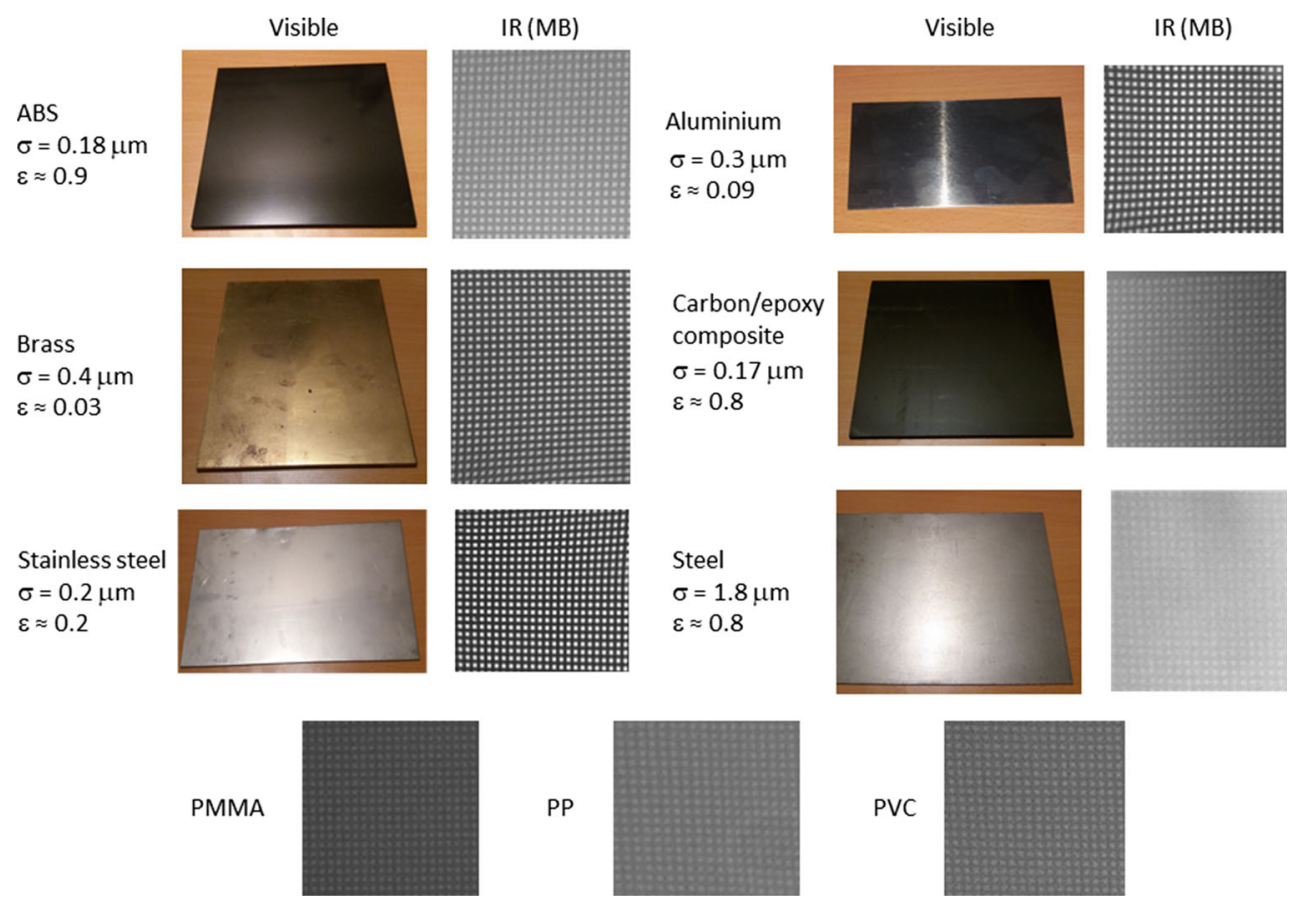

on otherwise transparent materials in the visible spectrum. In any case, their roughness was significantly lower than that of the other materials. Figure 12 shows pictures of the plates in the visible spectrum together with reflected grid images recorded by the MB camera. To calculate the $\lambda / \sigma$ ratios, the average wavelength of the detector sensitivity range was used. As can be seen in Table 1, the MB camera is sensitive in the band 7.5 to $14 \mu \mathrm{m}$, so the average of $10.7 \mu \mathrm{m}$ was used to calculate the Rayleigh ratio. Similarly, a mean sensitivity wavelength of $3.3 \mu \mathrm{m}$ was considered for the QD camera.

For all materials with the exception of the very rough steel plate, the reflected images provided enough contrast to perform good quality measurements. The $\lambda / \sigma$ ratios range from 13 to 60 , while the rough steel plate exhibits a $\lambda / \sigma$ ratio of 6 , confirming that the Rayleigh criterion is a good indicator of the feasibility of IR deflectometry. It can also be seen that the material's emissivity plays an important role in the quality of the reflected images. Indeed, assuming a zero transmittance, emissivity and reflectivity add up to one. Therefore, a high emissivity material will have a lower reflectance and so, exhibit a lower contrast reflected image. This is clear from Fig. 12 when comparing the low emissivity brass, stainless steel and aluminium to the ABS and carbon/epoxy composite. However, even when the emissivity is high, good enough contrast is obtained to perform measurements, though the phase noise is likely to be significantly higher. This will be studied in more detail in future work.

To investigate further the effect of roughness, a series of images were taken for an aluminium plate with both the MB and QD cameras. The plate 'as received' had a roughness of $0.4 \mu \mathrm{m}$ in one direction and $0.1 \mu \mathrm{m}$ in the other. P800, P240 and P120 sand paper grades were used to reach roughness values of $0.5 \mu \mathrm{m}, 1.4 \mu \mathrm{m}$ and $2.1 \mu \mathrm{m}$ respectively. The resulting images and associated histograms have been put together in Fig. 13.

For the MB camera, contrast significantly decreased from a $\lambda / \sigma$ of 21 to 7.7 , and all contrast was lost at $\lambda / \sigma=5$, which is consistent with the Rayleigh criterion. The images recorded with the QD camera clearly illustrate the advantage of imaging in the LW spectrum as all contrast is already lost for the $1.4 \mu \mathrm{m}$ roughness, though surprisingly, some contrast is visible for the $0.5 \mu \mathrm{m}$ roughness that provides a $\lambda / \sigma$ of 6.6 , which seems in violation of the Rayleigh criterion. However, the wavelength used for the QD camera is the average of its sensitivity band as reported in Table 1, so $3.3 \mu \mathrm{m}$. Since according to [2], a heated grid to $313 \mathrm{~K}$ (which is close to what is used here) has maximum emission at $9.3 \mu \mathrm{m}$, it is likely that only the upper range of the QD camera sensitivity band is active when recording the image. Replacing the average wavelength of $3.3 \mu \mathrm{m}$ by $5.1 \mu \mathrm{m}$, a $\lambda / \sigma$ of 10 is obtained, which is above the Rayleigh criterion.

The conclusions from this section are that the Rayleigh criterion is a very good indicator to check whether IR deflectometry can be used for a given surface, and also that LW IR cameras are to be preferred over MW cameras. With a typical LW IR camera, IR deflectometry can be used on a wide range of 'as manufactured' materials surfaces. Good enough images were obtained for roughness values up to $1.4 \mu \mathrm{m}$, compared to the $0.02 \mu \mathrm{m}$ roughness provided 


$$
\lambda / \sigma=21
$$
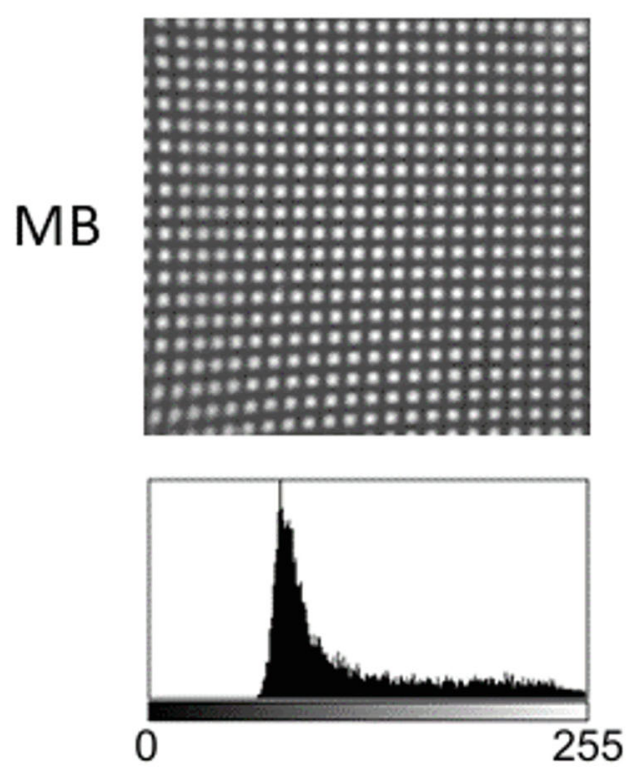

$\sigma=0.5 \mu \mathrm{m}$
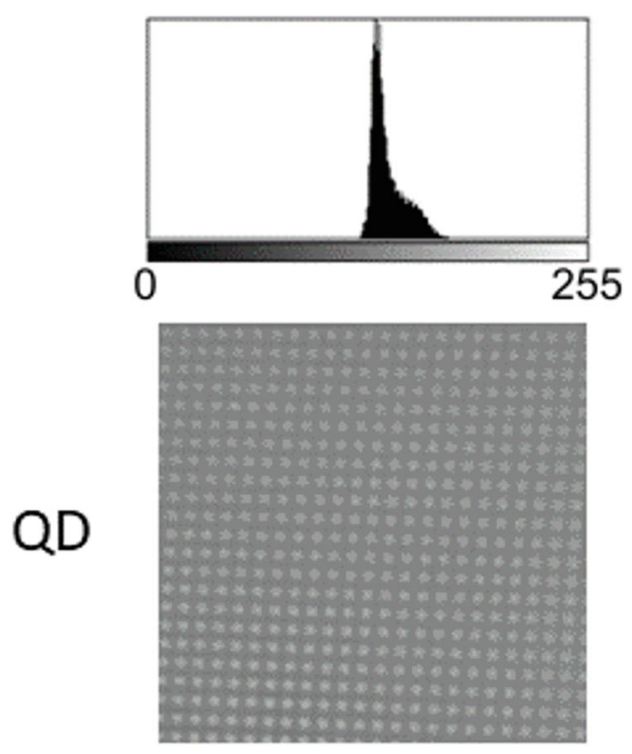

$$
\lambda / \sigma=6.6
$$

$\lambda / \sigma=7.7$
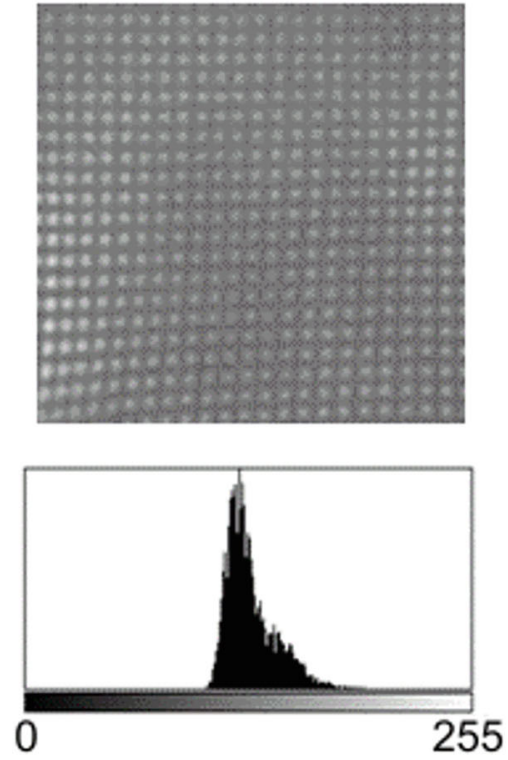

$\sigma=1.4 \mu \mathrm{m}$
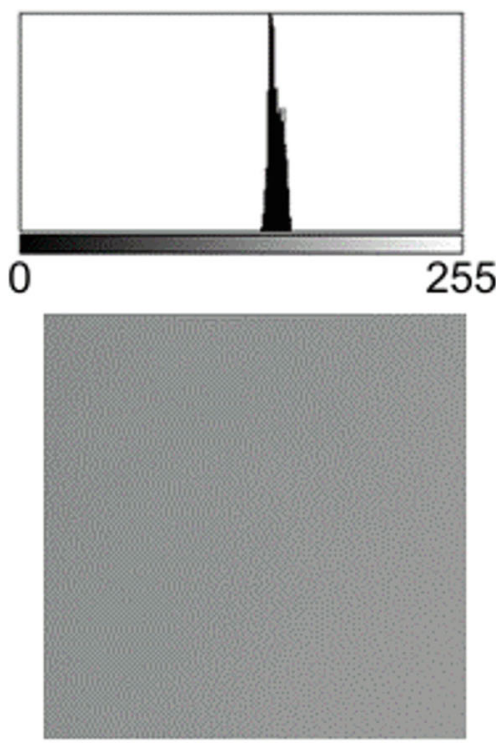

$\lambda / \sigma=2.4$ $\lambda / \sigma=5$
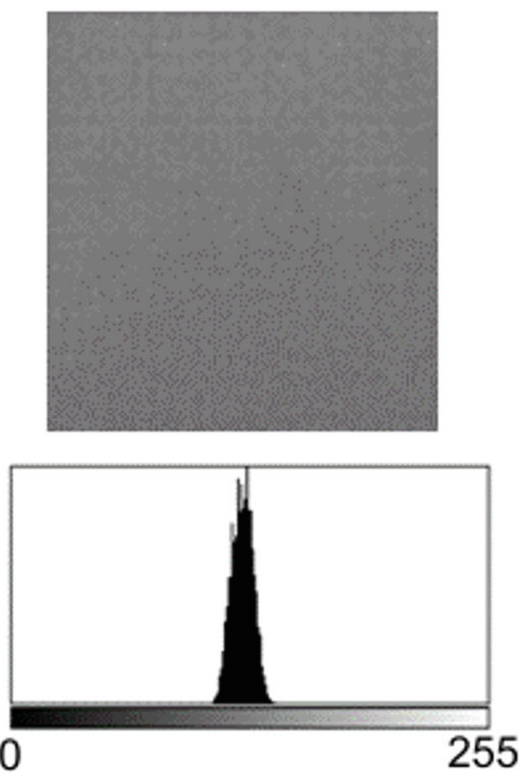

$\sigma=2.1 \mu \mathrm{m}$

Fig. 13 Images of reflected grid for the MB and QD cameras for an aluminium plate of increasing surface roughness $\sigma$

by the coatings described in [20] to enable imaging in the visible spectrum. Would the roughness be too high, as for the steel plate, mild sandpapering would be enough to bring it to a suitable level. It was also confirmed that low emissivity test materials exhibited better contrast of reflected image. The overall conclusion for this section is that IR deflectometry shows high potential to become an exciting new tool to measure material deformation or assess surface defects with very limited surface preparation if any.

\section{Bending Tests}

Finally, a series of plate bending tests were performed to validate the technique both against a finite element model of the test, and by extracting the material's elastic constants from the deformation maps using the Virtual Fields Method (VFM). The test configuration considered here is a thin rectangular aluminium plate pinned at three points and loaded centrally, as shown schematically in Fig. 14. The 


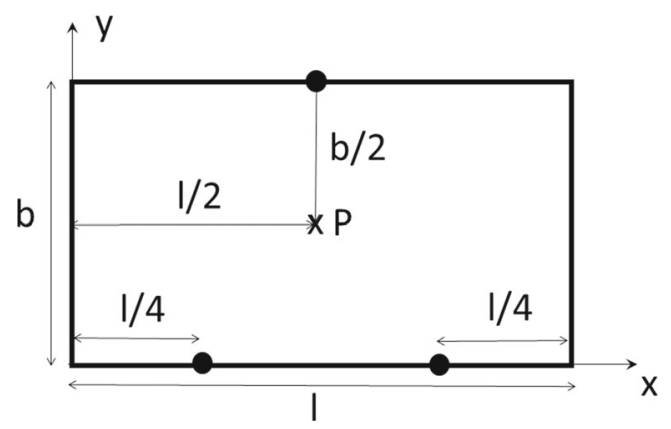

Fig. 14 Plate bending configuration: the black dots represent pinned supports while the central cross represents point loading in positive $\mathrm{Z}$ direction

camera was positioned as close as possible to the grid plate, and slightly tilted to fill up the camera field of view. This naturally created a small parallax effect. To mitigate this, the plate long axis was placed vertically and the camera rotated so that its $\mathrm{x}$-axis (with more pixels) was also aligned vertically. Three plates were tested, with dimensions shown in Table 2. The imaging distance was obtained using a tape measure and taken between the surface of test plate and the estimated optical centre of the imaging system, typically supposed to be located at the connection between the camera and the lens. It should be noted that a few millimetres uncertainty creates negligible errors with such a large imaging distance. The experimental setup is pictured in Fig. 15, with details in Fig. 16. The load was applied and measured through an in-house developed load cell as seen in Fig. 16. The grid pitch here was $7.18 \mathrm{~mm}$ in the $\mathrm{x}$-direction, and $7.10 \mathrm{~mm}$ in the $y$-direction. The uneven pitch is the result of the different resolutions of the printer in $\mathrm{x}$ and $\mathrm{y}$, as detailed in $[28,29]$, but the difference is small enough to prevent any parasitic fringes from appearing, as detailed in the section on grid quality and sampling. A mean grid pitch of $7.14 \mathrm{~mm}$ was selected for the grid processing. A sampling of 5 pixels per pitch $(\mathrm{N}=5)$ was used and the magnification adjusted to reduced the number of parasitic moiré fringes in the undersampled image, as per the protocol detailed in "Sampling and Grid Quality". The reason for moving to a larger pitch compared to the former $2 \mathrm{~mm}$ one was that the short lens only available to us did not allow to produce good enough imaging conditions at the magnification that was required for a $2 \mathrm{~mm}$ pitch, so both test and grid plates were scaled up.

Table 2 Dimensions of the tested plates and imaging distances

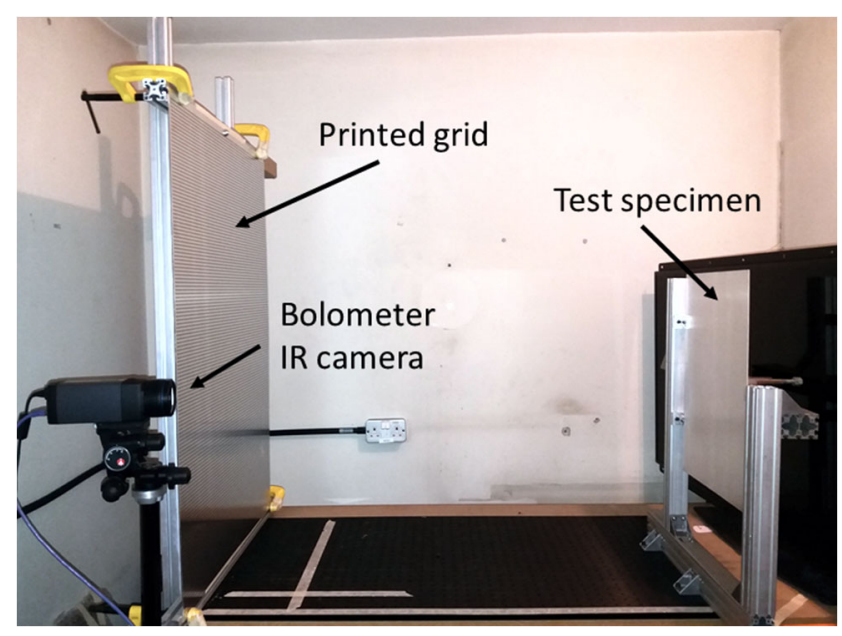

Fig. 15 Experimental setup

The raw images recorded by the MB camera are shown in Fig. 17 for Plate 1 (Table 2). As can be seen, the full spatial resolution of the camera has been used as only a few pixels are lost at the edges. This is not so true for Plate 3 which is much smaller and spatial resolution will be reduced there. On these images, the parallax effect related to the fact that the camera observes the plate from the grid target edge is evident. However, measuring the change of length between top and bottom edges (in pixels), a change of only $4 \%$ was found. This is small enough to prevent significant sampling issues in the phase calculations. The grid image quality tool detailed in "Sampling and Grid Quality" was used and no parasitic fringes were noticed, indicating that the effect of this parallax is negligible compared to the other sources of error. These images were first cropped to keep only the pixels corresponding to the test specimen. The phase difference was then calculated, unwrapped and scaled by $N / 2 \pi$ to provide slope in pixels. Again, only one data point per pitch was kept. Centred finite difference was then used to differentiate the slopes and obtain curvatures. It is worth noting that no smoothing of the slope field has been applied, and that the virtual strain gauge size (number of independent data points used to calculate the derivatives) was therefore 3 .

\section{Test Results and Comparison with FE Model}

Several tests have been conducted on the three plates in Table 2 but only one will be reported here for the

\begin{tabular}{lllll}
\hline & Length $(\mathrm{mm})$ & Width $(\mathrm{mm})$ & Thickness $(\mathrm{mm})$ & Imaging distance $(\mathrm{mm})$ \\
\hline Plate 1 & 451 & 320 & 1.5 & 1059 \\
Plate 2 & 421 & 315 & 1.5 & 1059 \\
Plate 3 & 339 & 216 & 1.5 & 1063
\end{tabular}




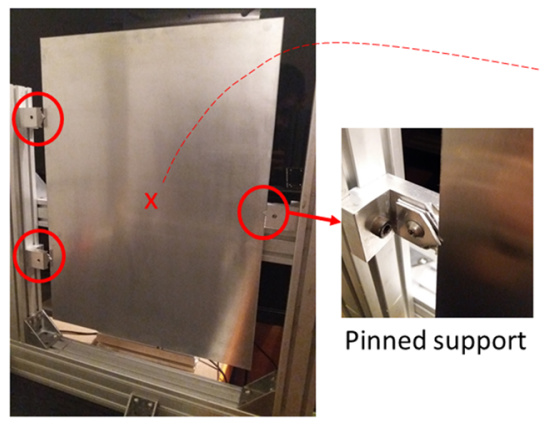

Three-point supported plate

Fig. 16 Experimental setup - details

purpose of comparison with a finite element model. Similar comparison can be obtained on the other tests, and the experimental images are available in the data archive attached to this paper. The test considered here is the first test on Plate 1 submitted to a central load of $15.1 \mathrm{~N}$. The slopes were obtained by phase subtraction between initial and loaded state images, unwrapping and scaling by $\mathrm{p} / 4 \mathrm{~h} \pi$ (where $\mathrm{p}$ is the reference grid pitch and $\mathrm{h}$ is the plate to grid distance) and only one data point per grid pitch has been kept so each data point is reasonably independent from the others. ${ }^{3}$ The curvatures were then calculated from the spatial derivatives of the slopes, and surface strains were derived by multiplying the curvatures by half the plate thickness. It is worth noting that no spatial smoothing has been employed either on the slope or derived curvatures, as is common with deflectometry that enjoys an excellent signal to noise ratio.

A finite element model of this test has been developed using ANSYS 17.1. A $200 \times 200$ mesh has been used with pinned boundary conditions for the support (i.e., fixed translations in the three directions, but free rotations) and a central point load of $15.1 \mathrm{~N}$. The elastic constants were $\mathrm{E}=69 \mathrm{GPa}$ for Young's modulus and $v=0.33$ for Poisson's ratio, typical values for aluminium. Surface element strains were then exported into Matlab R2017b to compare with the experiment. The three surface strain components are shown in Fig. 18 for both the experimental and simulated data.

One can see that even though the strains are quite small, they are two orders of magnitude higher than the measurement resolution which is typically on the order of $1 \mu$ strain for this configuration [34]. This explains why the random noise only moderately affects the data, even though no spatial smoothing has been employed. This illustrates the main advantages of deflectometry: very high sensitivity and only one differentiation needed to obtain curvatures /

\footnotetext{
${ }^{3}$ The WDFT algorithm uses $2 \mathrm{~N}-1$ pixels to calculate the phase, but because of the triangular window, the points away from the considered pixel have less influence on the measured phase
}

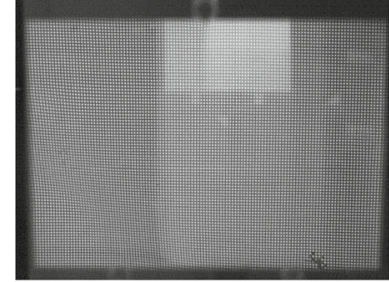

Unloaded state

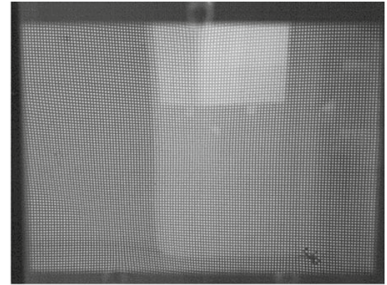

Loaded state (15.1 N)
Fig. 17 Images of the grid reflection in the unloaded and loaded states

strains. The quality of these data is largely above anything achievable through stereo digital image correlation which has a typical in-plane strain resolution around $100 \mu$ strain. It is interesting to note that some very local divergence exists between model and experiment close to the loading and support points, mostly due to the limitation in spatial resolution of the camera that cannot capture the fine details in these high strain concentration areas. But overall, the comparison is excellent. The next section looks at using these experimental data to identify the elastic constants of the plate as another validation, using the Virtual Fields Method.

\section{Virtual Fields Method}

The Virtual Fields method is a tool to identify material constitutive properties from full-field kinematic measurements [40]. It is based on the principle of virtual work expressing stress equilibrium. In the case of a linear elastic isotropic homogeneous (Love-Kirchhoff) thin plate in quasi-static

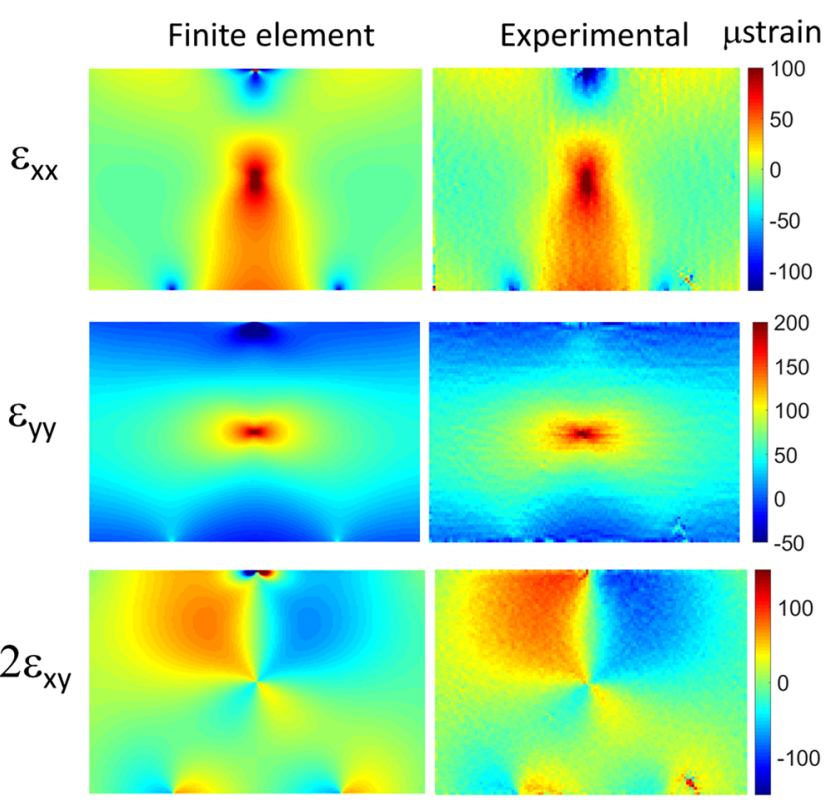

Fig. 18 Comparison between finite element and experimental surface strains 
bending under a set of point loads, the equation can be written as:

$$
\begin{aligned}
& -D_{x x} \int_{S}\left(k_{x} k_{x}^{*}+k_{y} k_{y}^{*}+\frac{1}{2} k_{s} k_{s}^{*}\right) d S-D_{x y} \int_{S}\left(k_{x} k_{y}^{*}+k_{y} k_{x}^{*}\right. \\
& \left.-\frac{1}{2} k_{s} k_{s}^{*}\right) d S+\sum_{i=1}^{n} P_{i} w_{i}^{*}=0
\end{aligned}
$$

where $D_{x x}$ and $D_{x y}$ are the two bending stiffness matrix components, $k_{x}, k_{y}$ and $k_{s}$ are the two normal and twist curvatures respectively (using Voigt's contracted notation where $\mathrm{s}$ denotes the engineering component of torsion), $k_{x}^{*}, k_{y}^{*}$ and $k_{s}^{*}$ are the related virtual curvature components deriving from the virtual deflection field $w^{*}$, and $P_{i}$ are the $\mathrm{n}$ point loads applied to the plate. The virtual deflection needs to be continuous and twice differentiable. Where the plate is simply supported and the reaction forces are unknown, $w^{*}$ needs to be zero at these points to prevent having unknown quantities in the equation; and where the plate is clamped, the virtual slopes also need to be zero. More details about the derivation of this equation can be found in [40]. The VFM has been used many times in the past on thin plates in bending [41-47] and the reader is also referred to these references for more details. Here, the VFM was used as a validation of the measurements as the elastic properties of the plate are known. As is customary with the VFM, Equation (2) can be discretized by approximating the continuous integrals. Introducing spatial averaging over the whole field of view using the overline notation, Equation (2) becomes:

$$
\begin{aligned}
& -D_{x x} S \overline{\left(k_{x} k_{x}^{*}+k_{y} k_{y}^{*}+\frac{1}{2} k_{s} k_{s}^{*}\right)} \\
& -D_{x y} S \overline{\left(k_{x} k_{y}^{*}+k_{y} k_{x}^{*}-\frac{1}{2} k_{s} k_{s}^{*}\right)}+\sum_{i=1}^{n} P_{i} w_{i}^{*}=0
\end{aligned}
$$

where $S$ is the surface of the plate. Since the material is linear elastic and isotropic, only two virtual fields need to be selected. As the supports are located only at two vertical locations, it is easy to define a constant curvature field that cancels out these reaction forces:

$w^{*(1)}=y(y-b)$

This corresponds to a constant virtual curvature field: $k_{x}^{*(1)}=0, k_{y}^{*(1)}=-2, k_{s}^{*(1)}=0$. The central point of coordinates $(l / 2, b / 2)$ exhibits a virtual displacement of $-b^{2} / 4$, providing the following equation:

$D_{x x} \overline{k_{y}}+D_{x y} \overline{k_{x}}=\frac{P b}{8 l}$
Table 3 Identification results

\begin{tabular}{clll}
\hline & Force $(\mathrm{N})$ & E $(\mathrm{GPa})$ & $v$ \\
\hline Plate 1 & & & \\
Test 1 & 15.1 & 70.6 & 0.30 \\
Test 2 & 17.4 & & 0.30 \\
Plate 2 & & 67.9 & \\
Test 1 & 20.4 & 66.4 & 0.33 \\
Test 2 & 25.5 & 62.2 & 0.32 \\
Plate 3 & 15.2 & 67.6 & 0.36 \\
Mean & - & 5.3 & 0.32 \\
C.V. $(\%)$ & & & 7.7 \\
\hline
\end{tabular}

For the second virtual field, since the supports are located at three different $\mathrm{x}$ coordinates, it is impossible to obtain a constant curvature field, whether $k_{x}^{*}$ or $k_{s}^{*}$. This is not a problem and a field like $w^{*}=(x-l / 4)(y-b)(x-3 l / 4)$ would work but for the sake of simplicity, it is possible to note that the problem is statically determinate here and symmetry together with equilibrium of external forces yields that the top reaction force is $-\mathrm{P} / 2$ and the two bottom ones are -P/4. So $w^{*(2)}=(x-l / 4)(x-3 l / 4)$ can be used in this case with an extra term coming from the virtual work of the top support and the central load. The virtual displacement of the top support point of coordinates $(1 / 2, b)$ is $-l^{2} / 16$, which also applies to the loading point. The associated virtual curvatures are $k_{x}^{*(1)}=-2, k_{y}^{*(1)}=0$, $k_{s}^{*(1)}=0$. So the second equation becomes:

$D_{x x} \overline{k_{x}}+D_{x y} \overline{k_{y}}=\frac{P l}{64 b}$

These virtual fields have first been tested on the FE simulated data reported in the previous section to validate them. They have then been applied to the data reported in "Test Results and Comparison with FE Model", for the three plates, some having been tested twice with an increased load. The results are reported in Table 3. Plate 1 is the largest in size and provides the best spatial resolution, as can be seen on the full images in the data repository. Not surprisingly, it also provides the best identification results, with $E$ and $v$ as expected for an aluminium alloy. Plate 2 provides a Young's modulus which is slightly too low, while Plate 3 see a much larger error on $E$. Errors can be caused by loss of some small amounts of data at the edges, the limited spatial resolution, the loss of Love-Kirchhoff assumption in the immediate vicinity of the supports and loading point as well as random noise in the images. Image deformation could be used to track these small errors, as in [48], but this is beyond the scope of the present paper. The results in Table 3 are enough to validate the measurement technique, which was the objective here. 


\section{Conclusion and Future Work}

This paper has shown the feasibility of deflectometry in the infrared spectrum to measure slope deformation at the surface of thin plates. This technique yields the classical advantages of deflectometry, high sensitivity and direct measurement of slopes, while addressing its main drawback, the need for a mirror-like surface. Imaging in the longwave infrared enables to use surfaces of roughness up to about $1.5 \mu \mathrm{m}$ without any surface preparation. It was found that the Rayleigh criterion was appropriate to establish whether IR deflectometry could be performed on a given surface. There are still a number of outstanding issues however.

- The first one is that infrared cameras and lenses have not been developed for photography but for thermography. As a consequence, the current spatial resolution of these cameras is still well below that of CCD or CMOS cameras with sensors readily available up to 29 Mpixels.

- Another problem is the cost. However, uncooled microbolometer cameras are much more affordable than quantum detector based cameras and are developing fast for the general public market (heat loss surveys, night imaging etc.). It is therefore reasonable to expect an increase in performance and a decrease of cost in the near future. In any case, the current camera has a list price of about $£ 15 \mathrm{k}$ and a spatial resolution of $640 \times 480$, which makes deflectometry with this camera reasonably affordable and provides adequate performance in the present case.

- Lenses represent another issue. Indeed, the present authors were unable to source infrared lenses with variable aperture, all lenses presenting quite a large aperture of $f / 1$ or $f / 3$. This is a drawback as for deflectometry, high depth of field is desirable. The use of a field aperture was explored by the present authors but with limited success.

- Finally, frame rate is also a limitation. Currently, visible light cameras can provide frame rates up to millions of frames per second [31]. This allows for timeresolved deflectometry even in the ultrasound range [34]. Bolometer-based infrared cameras are limited by their response time, here, $8 \mathrm{~ms}$, and the time for the active sensor element to cool down before another image can be taken. This means that frame rates are typically limited to less than $100 \mathrm{~Hz}(50 \mathrm{~Hz}$ with the current camera). To get higher frame rates, quantum detector cameras need to be employed. Currently, imaging up to $1 \mathrm{kHz}$ is possible with long wave cameras at resolutions around $500 \times 500$, but these cameras are much more expensive.
For IR deflectometry to become a widespread tool, it is essential that it can be applied to curved surfaces. Deflectometry in the visible range has been applied in the past but all implementations rely on temporal phase shifting from fringes displayed on an electronic screen, generally LCD [1]. It is however much more difficult to source a display screen that emits in the infrared, as explained in [21]. As part of this project, we tried to remove the screen of a mobile phone to check whether the OLEDs of the screen would emit enough infrared. Unfortunately, the screen protective plate is not fully IR transparent and the attempt to remove it to have direct illumination from the OLEDs failed. However, this attempt convinced us that OLEDS would provide sufficient illumination in the IR to perform screen-based temporal phase shifting. This will be explored in the near future. In any case, it would always be possible to use a static heated grid as that used in the present paper and mount it on a frame with translation stages to perform the phase shifting, as was already demonstrated in [21]. In this case, the curved deflectometry setup presented in [49] could easily be implemented for quasi-static measurements. The depth-of-field issue mentioned above may however limit this to moderately curved surfaces. For harmonic vibrations, it would be possible to synchronize the phase shifting with the excitation to perform strobe-like phase shifting. And for time-resolved measurements, a high-speed camera would need to be used, together with dual grid pattern ${ }^{4}$ such that its reflection is regular to enable spatial phase shifting. The current authors believe that there is a lot of scope in the future for this method which has the potential to become a new tool for the experimentalist. Future work will be conducted in that direction.

Acknowledgments The authors are grateful to Dr Devlin Hayduke from the Materials Sciences Corporation, Horsham, PA, USA for suggesting the idea, and to Dr Yves Surrel for useful discussions on deflectometry and grid printing.

Funding Horea Toniuc acknowledges funding through the Excel Southampton Internship Programme.

Data provision All data supporting this study are openly available from the University of Southampton repository at https://doi.org/10. 5258/SOTON/D0805.

Open Access This article is distributed under the terms of the Creative Commons Attribution 4.0 International License (http://creativecommons. org/licenses/by/4.0/), which permits unrestricted use, distribution, and reproduction in any medium, provided you give appropriate credit to the original author(s) and the source, provide a link to the Creative Commons license, and indicate if changes were made.

\footnotetext{
${ }^{4}$ If the reflection of a regular grid on a non-flat surface is irregular, it is possible, after calibration, to calculate which (irregular) grid should be displayed so that its image is regular. This is what is called here the 'dual' grid.
} 


\section{References}

1. Balzer J, Werling S (2010) Principles of shape from specular reflection. Measurement 43(10):1305-1317

2. Horbach J, Kammel S (2005) Deflectometric inspection of diffuse surfaces in the far-infrared spectrum. In: Electronic imaging 2005, vol. 5679, SPIE

3. Huang L, Seng Ng C, Krishna Asundi A (2012) Fast full-field outof-plane deformation measurement using fringe reflectometry. Opt Lasers Eng 50(4):529-533

4. Arnal L, Solanes JE, Molina J, Tornero J (2017) Detecting dings and dents on specular car body surfaces based on optical flow. J Manuf Syst 45:306-321

5. Ligtenberg F (1954) A new experimental method for the determination of moments in small slab models. Proceedings of SESA XII 2:83-98

6. Chiang FP, Treiber J (1970) A note on Ligtenberg's reflective moiré method - technical note offers an additional improvement to the optical arrangement whereby the sensitivity of the method can be changed at will. Exp Mech 10(9):537-538

7. Kafri O, Livnat A (1981) Reflective surface analysis using moiré deflectometry. Appl Opt 20(18):3098-3100

8. Kao TY, Chiang FP (1982) Family of grating techniques of slope and curvature measurements for static and dynamic flexure of plates. Opt Eng 21(4):214721-214721

9. Ritter R (1982) Reflection moiré methods for plate bending studies. Opt Eng 21(4):663-671

10. Asundi A (1994) Novel techniques in reflection moiré. Exp Mech 34(3):230-242

11. Surrel Y, Fournier N, Grédiac M., Paris PA (1999) Phase-stepped deflectometry applied to shape measurement of bent plates. Exp Mech 39(1):66-70

12. Devivier C, Pierron F, Wisnom M (2012) Damage detection in composite materials using full-field slope measurements. Compos A: Appl Sci Manuf 43(10):1650-1666

13. Periasamy C, Tippur HV (2013) A full-field reflection-mode digital gradient sensing method for measuring orthogonal slopes and curvatures of thin structures. Meas Sci Technol 24:2

14. Ri S, Fujigaki M, Morimoto Y (2010) Sampling moiré method for accurate small deformation distribution measurement. Exp Mech 50(4):501-508

15. Surrel Y (1994) Moiré and grid methods: a signal-processing approach. In: Proceedings of SPIE - The international society for optical engineering, vol 2342, pp 118-127

16. Grédiac M, Sur F, Blaysat B (2016) The grid method for inplane displacement and strain measurement: a review and analysis. Strain 52(3):205-243

17. Jailin C (2018) Full field modal measurement with a single standard camera. Opt Lasers Eng 107:265-272

18. Grédiac M, Blaysat B, Sur F (2017) A critical comparison of some metrological parameters characterizing local digital image correlation and grid method. Exp Mech 57(6):871-903

19. Ogilvy J (1991) Theory of wave scattering from random rough surfaces. CRC Press, Boca Raton

20. Kim J-H, Pierron F, Grédiac M, Wisnom M (2007) A procedure for producing reflective coatings on plates to be used for fullfield slope measurements by a deflectometry technique. Strain 43(2):138-144

21. Höfer S, Burke J, Heizmann M (2016) Infrared deflectometry for the inspection of diffusely specular surfaces. Adv Opt Technol 5(5-6):377

22. Sarosi Z, Knapp W, Kunz A, Wegener K (2010). Detection of surface defects on sheet metal parts by using one-shot deflectom- etry in the infrared range. In: Proceedings of InfraMation 2010 conference, 8-10 November 2010 in Las Vegas, Nevada, USA

23. Höfer SWS, Beyerer J (2013) Thermal pattern generation for infrared deflectometry. In: AMA Conferences 2013 - SENSOR 2013, OPTO 2013, IRS 22013

24. Su T, Wang S, Parks RE, Su P, Burge JH (2013) Measuring rough optical surfaces using scanning long-wave optical test system. 1 . principle and implementation. Appl Opt 52(29):7117-7126

25. Data sheet for the FLIR SC7600 infrared camera. https://support. flir.com/DsDownload/Assets/66101-0101-en-US.html, last accessed 04/09/2018

26. Data sheet of the FLIR A6550 $25^{\circ}$ infrared camera. https:// support.flir.com/DsDownload/Assets/55001-0302-en-US.html, last accessed 04/09/2018

27. Riedl M (2001) Optical design fundamentals for infrared systems, 2nd edn. SPIE Press, Bellingham

28. Grid printing procedure part I. http://photodyn.org/wp-content/ uploads/2016/03/Report_UniversityPrintCentre.pdf, last accessed $04 / 09 / 2018$

29. Grid printing procedure part II. http://photodyn.org/wp-content/ uploads/2016/03/Report_UniversityPrintCentre_2.pdf, last accessed 04/09/2018

30. Wire wound flexible heaters from Holroyd Components Ltd. http://www.holroydcomponents.com/products/wire-wound-flexibleheaters/, last accessed 04/09/2018

31. Pierron F, Cheriguene R, Forquin P, Moulart R, Rossi M, Sutton M (2011) Performances and limitations of three ultra high-speed imaging cameras for full-field deformation measurements. Appl Mech Mater 70:81-86. Trans Tech Publications Ltd

32. Matchid software suite. http://www.matchid.eu, last accessed 07/09/2018

33. Sur F, Grédiac M (2014) Sensor noise modeling by stacking pseudo-periodic grid images affected by vibrations. IEEE Signal Process Lett 21(4):432-436

34. Devivier C, Pierron F, Glynne-Jones P, Hill M (2016) Timeresolved full-field imaging of ultrasonic lamb waves using deflectometry. Exp Mech 56(3):345-357

35. Morgan R, Reid R, Baker A, Lucero B, Bernardin J (2017) Emissivity measurements of additively manufactured materials, tech. rep., Los Alamos National Lab. (LANL), Los Alamos, NM (United States)

36. Wolfe W (1965) Handbook of military infrared technology, tech. rep. Michigan University Ann Arbor

37. Öhman C (1999) Emittansmätningar med AGEMA E-Box, teknisk rapport, tech. rep., AGEMA Technical Report (FLIR Systems AB): Stockholm

38. Bramson M (2013) Infrared radiation: a handbook for applications. Springer Science \& Business Media

39. Gomez-Vasquez S, Brown A, Hubbard J, Ramirez C, Dodd A (2011) Carbon fiber composite characterization in adverse thermal environments., tech. rep. Sandia National Laboratories

40. Pierron F, Grédiac M. (2012) The virtual fields method. Extracting constitutive mechanical parameters from full-field deformation measurements, Springer, New-York

41. Grédiac M, Vautrin A (1990) A new method for determination of bending rigidities of thin anisotropic plates. J Appl Mech-Trans ASME 57(4):964-968

42. Grédiac M, Fournier N, Paris PA, Surrel Y (1998) Direct identification of elastic constants of anisotropic plates by modal analysis: Experimental results. J Sound Vib 210(5):643-659

43. Avril S, Grédiac M, Pierron F, Surrel Y, Toussaint E (2004) Deflectometry and virtual fields for identification of static plate bending stiffnesses. Annual SEM conference 
44. Kim J-H, Pierron F, Wisnom M, Avril S (2009) Local stiffness reduction in impacted composite plates from full-field measurements. Compos A: Appl Sci Manuf 40(12):1961-1974

45. Giraudeau A, Pierron F, Guo B (2010) An alternative to modal analysis for material stiffness and damping identification from vibrating plates. J Sound Vib 329(10):1653-1672

46. Devivier C, Pierron F, Wisnom M (2013) Impact damage detection in composite plates using deflectometry and the virtual fields method. Compos A: Appl Sci Manuf 48:201-218

47. Xavier J, Belini U, Pierron F, Morais J, Lousada J, Tomazello M (2013) Characterisation of bending stiffness of MDF from full-field slope measurements. Wood Sci Technol 47(2):423-441
48. Wang P, Pierron F, Rossi M, Lava P, Thomsen OT (2016) Optimised experimental characterisation of polymeric foam material using dic and the virtual fields method. Strain 52(1): $59-79$

49. Surrel Y, Pierron F (2019) Deflectometry on curved surfaces, vol. 3 of conference proceedings of the Society for Experimental Mechanics series. Springer 2018 Conference ed.

Publisher's Note Springer Nature remains neutral with regard to jurisdictional claims in published maps and institutional affiliations. 